\title{
LAS POLIITICAS SOBRE EDUCACIÓN BÁSICA EN AMÉRICA LATINA: LAS PERSPECTIVAS DE LOS ORGANISMOS INTERNACIONALES
}

\author{
Jorge M. Gorostiaga* \\ Universidad Nacional de San Martín, Buenos Aires - Argentina \\ Lucía Ferrere** \\ Universidad de Buenos Aires, Buenos Aires - Argentina
}

RESUMEN: Este artículo busca contribuir a la comprensión de cómo se estructura actualmente la agenda de reforma educativa en América Latina. Uno de los principales instrumentos que utilizan las organizaciones internacionales en la definición de la agenda y del discurso de las políticas sobre educación es la difusión de documentos de diagnóstico y propuestas de mejoras. El artículo analiza los posicionamientos de un conjunto de documentos producidos y/o promovidos, durante el período 2012-2014, por algunos de los principales organismos que participan en la cooperación técnica y financiera en América Latina. Para ello compara las perspectivas de análisis, los balances, los desafíos y las recomendaciones que los documentos presentan sobre las políticas de educación básica implementadas en la región desde comienzos de la década de 2000.

Palabras clave: Reforma educativa. Organismos internacionales. América Latina.

\section{AS POĹTICAS SOBRE EDUCAC̣ÃO BÁSICA NA AMÉRICA LATINA: AS PERSPECTIVAS DAS ORGANIZAC̄̃̃ES INTERNACIONAIS}

RESUMO: Este artigo procura contribuir para a compreensão de como se estrutura a agenda de reforma da educação na região da América Latina atualmente. Um dos principais instrumentos utilizados pelas instituições educacionais na definição da agenda e no discurso das políticas sobre educação é a divulgação de documentos de diagnóstico e de propostas de melhorias. O artigo analisa as posições de um conjunto de documentos produzidos e/ ou promovidos, durante o período de 2012-2014, por algumas das principais organizações envolvidas na cooperação técnica e financeira na América

"PhD Análisis Social y Comparado en Educación, University of Pittsburgh. Profesor y director de la Maestría en Gestión Educativa de la Universidad Nacional de San Martín. Profesor de Posgrado del Área Educación de la Univ. Torcuato Di Tella Investigador del CONICET. E-mail:<jorgegoros@gmail.com> .

"Licenciada en Sociología y estudiante de la Maestría en Investigación en Ciencias Sociales de la Universidad de Buenos Aires. Es Jefe de Trabajos Prácticos en la Facultad de Derecho de la Universidad Nacional de Lomas de Zamora. E-mail:<luferrere@gmail.com>. 
Latina. Para isso, compara as perspectivas de análise, os balanços, os desafios e as recomendações que os documentos apresentam sobre as políticas de educação básica implementadas na região desde o início da década de 2000. Palavras-chave: Reforma educacional. Organizações internacionais. América Latina.

\section{POLICIES ON BASIC EDUCATION IN LATIN AMERICA: THE PERSPECTIVES OF INTERNATIONAL ORGANIZATIONS}

ABSTRACT: This article seeks to contribute to the understanding of how education reform agenda in the Latin America region is currently structured. One of the main instruments used by educational organizations in setting the agenda and the discourse on education policy is the dissemination of documents that provide diagnosis and suggestions for improvements. The article analyzes the positions of a set of documents produced and/or promoted, during the 2012-2014 period, by some of the major international organizations involved in technical and financial cooperation in Latin America. For that purpose, it compares the perspectives of analysis, balances, challenges and recommendations that the documents present on the basic education policies implemented in the region since the early 2000s. Keywords: Education reform. International organizations. Latin America.

\section{INTRODUCCIÓN}

Los organismos internacionales son actores de peso en el escenario latinoamericano de las reformas educativas (POGGI, 2014; KRAWCZYK, 2002). Uno de los principales instrumentos que utilizan en la definición de la agenda y del discurso de las políticas sobre educación es la difusión de documentos de diagnóstico y propuestas de mejoras. Sin desdeñar el argumento sobre la conformación de una agenda global de reforma educativa en la que los organismos internacionales (interactuando con otros actores) juegan un rol decisivo (RAMBLA, 2013), este artículo pone el acento en identificar las diferencias en los posicionamientos textuales, en la línea de estudios previos (por ejemplo, GOROSTIAGA; TELLO, 2011) sobre las perspectivas discursivas acerca de las reformas educativas en América Latina.

Esta región presenta, desde comienzos de la década de 2000, un panorama políticamente heterogéneo en el que han convivido, por ejemplo, regímenes autodenominados socialistas (como la Venezuela chavista) con gobiernos de tinte conservador (como el de Uribe en Colombia), en un proceso de "compleja y ambigua repolitización" a través de una diversidad de liderazgos 
(ORJUELA, 2012, p. 199). Las reformas educativas que han tenido lugar en los distintos países, manifestadas en algunos casos en nuevas leyes de educación, han reflejado dicha heterogeneidad (PULIDO CHAVES, 2010; SAFORCADA; VASSILIADES, 2011). Uno de los elementos novedosos es el énfasis, al menos a nivel normativo, en la educación intercultural. A nivel más general, puede señalarse cómo, después de la década de 1990 -marcada por un fuerte movimiento de transformación de los sistemas educativos en el marco de una reconfiguración neoliberal del Estado- asistimos en la región en los últimos años a un escenario "de negociaciones de significado y de lucha cultural por definir las orientaciones político-educativas" (SAFORCADA; VASSILIADES, 2011, p. 302).

Este artículo analiza el posicionamiento de un conjunto de documentos producidos, durante el período 2012-2014, por algunas de las principales agencias que participan en la cooperación técnica y financiera en América Latina. El objetivo del trabajo es caracterizar el discurso de los organismos internacionales en términos de los balances sobre las políticas implementadas en los últimos 15 años y las recomendaciones que se derivan de ellos en el ámbito de la educación básica (desde el nivel inicial al secundario). De esta manera, buscamos contribuir a la comprensión de cómo se estructura actualmente la agenda de reforma educativa en la región.

La búsqueda de documentos se orientó a textos que fueran producidos y/o promovidos por los principales organismos que la literatura (OREJA CERRUTI; VIOR, 2016; KRAWCZYK, 2002; RODRÍGUEZ-GÓMEZ; ALCÁNTARA, 2001) reconoce como los que, tradicionalmente y en particular en la década de 2000, han tenido protagonismo en la región a nivel educativo. No hemos identificado documentos de la Organización de Estados Americanos, la Comisión Económica para América Latina y el Caribe ni del Banco Mundial producidos en este período para el sector educación de la región como un todo. ${ }^{1}$ En la selección de los textos, se buscó incluir la mayor cantidad de organismos posibles privilegiando los textos que brindaran un panorama de las políticas a nivel regional y que formularan recomendaciones hacia el futuro. La lista de los documentos seleccionados, en los que, en algunos casos, se involucra más de una organización, es la siguiente:

a) CABROL, Marcelo; SZÉKELY, Miguel. Educación para la transformación. New York: Banco Interamericano de Desarrollo, 2012. cap. Introducción, p. v-xxvii; cap. Conclusión, p. 383-387.

b) GAJARDO, Marcela. La educación tras dos décadas de cambio: ¿Qué 
hemos aprendido? ¿Qué debemos transformar?. Santiago de Chile: PREAL Publicaciones, 2012. (Serie Documentos, n. 65.)²

Documento preparado parala Organización de Estados Iberoamericanos para la Educación, la Ciencia y la Cultura.

c) BELLEI, C. (Coord.). Situación Educativa de América Latina y el Caribe: hacia la educación de calidad para todos al 2015. Santiago de Chile: Oficina Regional de Educación para América Latina y el Caribe (OREALC/UNESCO), 2013.

d) POGGI, M. La educación en América Latina: logros y desafíos pendientes: documento básico. Buenos Aires: Santillana, 2014.

Documento básico del X Foro Latinoamericano de Educación.

e) ORGANIZACIÓN DE ESTADOS IBEROAMERICANOS PARA LA EDUCACIÓN, LA CIENCIA Y LA CULTURA. Miradas sobre la educación en Iberoamérica: avances en las Metas Educativas 2021. Madrid: OEI, 2014.

f) ORGANIZACIÓN DE ESTADOS IBEROAMERICANOS PARA LA EDUCACIÓN, LA CIENCIA Y LA CULTURA. Miradas sobre la educación en Iberoamérica: desarrollo profesional docente. Madrid: OEI, 2013.

En este trabajo utilizamos el método del análisis temático (GUEST; MACQUEEN; NAMEY, 2011) para identificar, caracterizar y comparar las perspectivas de análisis, los balances sobre las políticas de educación básica implementadas en la región desde comienzos de la década de 2000, los desafíos actuales y las recomendaciones hacia el futuro que los documentos presentan. Este tipo de abordaje pone el acento en describir las ideas implícitas y explícitas que se desprenden de los datos disponibles. A la vez, llevamos adelante un análisis textual que busca representar los argumentos de cada documento en sus propios términos, a partir del cual identifica puntos de contacto y diferencias entre ellos. En este sentido, adoptamos un enfoque perspectivista que enfatiza cómo cada posicionamiento textual expresa una determinada manera de percibir y representar el fenómeno abordado (MARCELO, 2014).

El artículo se organiza en dos secciones principales. En la primera parte, desarrollamos un breve marco conceptual sobre la nuestra concepción de la política educativa y del rol que juegan los OOII en ella. La segunda parte está dedicada al análisis de los documentos seleccionados. 


\section{MARCO CONCEPTUAL}

En este estudio partimos de una visión procesual de las políticas educativas, la cual supone enfatizar el dinamismo y el conflicto inherente a todo el ciclo o trayectoria de una política educativa (NUDZOR, 2009). En tales procesos, no sólo la acción del Estado-nación juega un rol fundamental, sino también agentes y factores que "por arriba y por abajo" participan de la regulación del sistema educativo, incluyendo la influencia de redes e imaginarios globales (RIZVI; LINGARD, 2013), organizaciones de la sociedad civil (BURCH, 2009) y de actores educativos a niveles meso y micro (BALL, 2011). A la vez, adoptamos una posición constructivista sobre el rol de las ideas en la formulación de las políticas educativas, de acuerdo con la cual elementos como la persuasión, la selectividad discursiva y la construcción de significado asumen un lugar central (VERGER, 2014). ${ }^{3}$ La posición constructivista sostiene que determinadas ideas, a través de procesos disputados de difusión e interpretación en los que se ponen en juego capitales simbólicos e institucionales, se convierten en políticamente influyentes no por su calidad o rigor intrínsecos, sino por la forma como son promovidas y presentadas a los decisores de política.

Tanto a nivel global como regional, los organismos internacionales -tanto los de carácter financiero como aquellos dedicados a la generación de consensos o a la asistencia técnicason actores fundamentales en el escenario actual de las reformas educativas (POGGI, 2014; RAMBLA, 2013). Uno de los principales instrumentos que utilizan en la definición de la agenda y del discurso de las políticas sobre educación es la difusión de documentos de diagnóstico y propuestas de mejoras (KRAWCZYK, 2002). Por otra parte, aunque suelen compartir aspectos importantes del diagnóstico y de las recomendaciones de política (KRAWCZYK, 2002; SANCHEZ CERÓN, 2001), también exhiben notorias discrepancias, siendo la más clásica la diferencia de orientación por parte de la UNESCO y el Banco Mundial desde su creación luego de la Segunda Guerra Mundial (MUNDY, 1998).

En América Latina, desdela activa promoción dela planificación educativa por parte de la OEA y UNESCO en la década de 1960, y pasando por el influyente documento de CEPAL-UNESCO (1992) y el protagonismo del Banco Mundial y el Banco Interamericano de Desarrollo (BID) en las reformas de la década de 1990 (KROTSCH, 2001; SÁNCHEZ CERÓN, 2001), los organismos internacionales han mantenido una significativa presencia en los procesos de discusión 
y formulación de políticas educativas. Iniciativas como el Proyecto Principal de Educación - motorizado por la Oficina Regional de Educación para América Latina y el Caribe (OREALC/UNESCO)la Educación para Todos -impulsada a nivel global por la UNESCO y el Banco Mundial-y las Metas 2021 -auspiciadas por la Organización de Estados Iberoamericanos (OEI) - han comprometido a los países de la región en esfuerzos de cambio de relativa amplitud durante las últimas décadas. Aun cuando ha ido variando el peso de las distintas agencias en estos procesos, la tendencia ha sido a un aumento de la cantidad de organizaciones, sobre todo las de nivel regional. En este sentido se destaca la aparición del Programa de Promoción de la Reforma Educativa (PREAL) ${ }^{4}$ en 1995 y de la oficina Buenos Aires del IIPE-UNESCO en 1998, así como el fortalecimiento de la OEI desde comienzos de la década de 2000. Por otra parte, también es destacable el ingreso de algunos países de la región en la Organización para la Cooperación y el Desarrollo Económicos (OCDE) y en el Programa PISA a partir del año 2000.

El análisis de las propuestas de distintos organismos internacionales y de su influencia sobre las políticas educativas nacionales ha sido objeto de numerosos trabajos en América Latina, particularmente desde perspectivas críticas que han denunciado la promoción de medidas más preocupadas por la eficiencia y la reducción de costos que por la mejora de la calidad y la democratización de los sistemas educativos (entre otros, BONAL, 2002; CORAGGIO; TORRES, 1997; FELDFEBER, 2007; OREJA CERRUTI; VIOR, 2016; PEREIRA; PRONKO, 2015; RODRÍGUEZ-GÓMEZ; ALCÁNTARA, 2001; SÁNCHEZ CERÓN, 2001; TELLO; MAINARDES, 2015). El trabajo de Krawczyk (2002) es uno de los principales antecedentes para nuestro estudio. ${ }^{5} \mathrm{Si}$ bien reconoce la ausencia de una posición monolítica entre los organismos internacionales, el análisis se orienta a identificar las principales coincidencias y los elementos preponderantes en el discurso de estas agencias sobre la reforma educativa en América Latina. Así, Krawczyk (2002) destaca que, a través de sus documentos, se defendió la concertación de políticas entre diferentes grupos sociales; el fortalecimiento de la participación de los sectores privados; la implementación de un nuevo modelo de gestión descentralizada del sistema escolar y de gran autonomía financiera, administrativa y pedagógica de las escuelas; y un uso más eficiente de los recursos existentes (sin cuestionar su insuficiencia). La autora finaliza su análisis remarcando la adhesión de este discurso a un modelo (capitalista) de desarrollo que limita fuertemente las posibilidades 
de transformaciones educativas que contribuyan a sociedades más igualitarias, y calificando a las propuestas de política de los organismos internacionales como "fragmentadas, contradictorias, mínimas, puntuales, además de privatizantes” (KRAWCZYK, 2002, p. 655).

\section{ANÁLISIS DE LOS DOCUMENTOS}

Esta sección presenta una descripción del posicionamiento de cada uno de los documentos seleccionados, organizada en cuatro dimensiones: perspectiva de análisis, balance de las políticas implementadas en los últimos 10/15 años, desafíos actuales y recomendaciones hacia el futuro. Seguidamente se discuten los puntos de convergencia y divergencia de los textos.

El documento del BID (CABROL; SZÉKELY, 2012) ${ }^{6}$ fue organizado por un funcionario y un consultor de la División Educación del organismo, a la vez autores de los dos capítulos que analizamos. El texto se centra en los avances de los sistemas educativos de América Latina desde, aproximadamente, el año 2000 y su prioridad en la agenda política. Además, discute los desafíos pendientes para lograr intervenciones en pos de la equidad y la calidad educativa, y se plantea contribuir a la propuesta de un nuevo modelo educativo pertinente al contexto de principios de la década de 2010 y sus necesidades. ${ }^{7}$

Cabrol y Székely (2012, p. v) parten de la idea de la educación como "motor imprescindible de progreso". Si bien plantean que en la educación convergen impactos económicos, políticos y de gobernabilidad, el foco principal está puesto en el aspecto de la adaptación a la economía del conocimiento: "sin educación difícilmente la región podrá transformarse y adaptarse para aprovechar las oportunidades que ofrece la era del conocimiento del nuevo milenio" (CABROL; SZÉKELY, 2012, p. v-vi). En esta línea, se señala la necesidad de un cambio de raíz de los sistemas educativos de la región, "una transformación radical del servicio educativo en varias dimensiones simultáneamente" (CABROL; SZÉKELY, 2012, p. 384). Un supuesto fundamental en el planteo de los autores es que es posible identificar intervenciones "exitosas" (que todos los actores sociales reconocerían como tales) para ser escaladas al conjunto del sistema educativo. En este sentido, puede hablarse de una visión tecnocrática que tiende a ignorar el carácter político y conflictivo de toda política educativa.

En la evaluación general de las políticas, el texto argumenta que los logros en la ampliación de la cobertura en la escolaridad y en la equidad de género han sido acompañados por la persistencia 
de "grandes deudas" respecto de la calidad educativa y de la contribución a una mayor igualdad social. Así, se destaca que existen grandes brechas de calidad educativa por nivel socioeconómico y en detrimento de los estudiantes indígenas y de zonas rurales. A ese diagnóstico le agrega que ha habido un limitado aprovechamiento de los avances tecnológicos.

A un balance predominantemente negativo de las políticas, los autores contraponen el rescate de "experiencias exitosas" y "alternativas novedosas que se han identificado paraimpulsarla calidad dela educación" apoyadas y ejecutadas por el BID (CABROL; SZÉKELY, 2012, p. ix). Se trata de tres programas que buscaron incidir, respectivamente, en la primera infancia en Ecuador; en la selección, formación y prácticas docentes (a través de las versiones argentina, chilena y peruana del controvertido Teach for America); y en la implementación de técnicas innovadoras para la enseñanza de la matemática en Argentina. Estas intervenciones, se señala, además de revertir las desventajas con las que ingresan al sistema los sectores más desfavorecidos, han logrado "avances sorprendentes" en términos de aprendizaje.

Los desafíos en el siglo XXI (en el siglo XX, se aclara, el foco fue la expansión de la cobertura de la escolaridad básica, hasta los 14/15 años) abarcan tres prioridades centrales: el mejoramiento de la calidad de la educación básica (logros de aprendizaje medibles); la construcción de ciudadanía (de la mano de la ampliación de la cantidad de países con regímenes democráticos); y la cobertura del nivel secundario alto (15-18 años), donde existe una alta deserción debido en parte a la falta de pertinencia de la propuesta educativa.

Las recomendaciones de los capítulos analizados responden a una visión que introduce componentes técnicos para una mayor eficiencia, combinada con una preocupación por romper con la reproducción de desigualdades educativas. Se postula que la política educativa debería estructurarse sobre dos elementos:

a) un consenso político y social amplio sobre la necesidad de que toda la sociedad asuma la tarea del mejoramiento de la educación;

b) la construcción de cuatro pilares:

- la definición del perfil de egreso-competencias y habilidades- en los diferentes niveles, del cual se derivaría el perfil requerido de docente y de directivo escolar;

- la construcción de un nuevo modelo educativo centrado en los alumnos en función de lograr su perfil de egreso, en el que "los docentes se transformen de catedráticos en acompañantes y facilitadores en el proceso de aprendizaje" (CABROL; SZEKELY, 2012, p. 385); 
- un conjunto de insumos necesarios para que el modelo educativo modernizado genere el perfil de egreso, que incluye, entre otros elementos, la selección y formación docente, la infraestructura y un modelo de gestión escolar con alto grado de autonomía;

- mecanismos de evaluación, monitoreo y medición que permiten verificar en qué medida el sistema como un todo y cada centro educativo en particular se acercan a la generación del perfil de egreso. Se sugiere poner en práctica mecanismos de certificación de docentes y directivos, así como de la calidad en infraestructura y equipamiento por centro escolar; también el uso de pruebas estandarizadas como PISA, TIMMS y otras para medir los avances en logros académicos.

A su vez, se pone el acento en la prioridad de atender a lo que se consideran cuestiones clave para el mejoramiento de la calidad y la equidad: la atención temprana y preescolar; la formación y las condiciones laborales de los docentes; y la disponibilidad de tecnologías, así como de metodologías y contenidos actualizados para optimizar los procesos pedagógicos.

El segundo documento es un texto (GAJARDO, 2012) preparado para la OEI en el marco de las actividades del proyecto Metas 2021 y publicado por el PREAL en su sitio web. Desde su título _"La educación tras dos décadas de cambio. ¿Qué hemos aprendido? ¿Qué debemos transformar?”-, queda explícito el propósito de un balance general que permita formular recomendaciones de cambio. Su foco está puesto en la calidad del aprendizaje, junto con consideraciones respecto a la equidad y la eficiencia.

Aunque no adopta una visión puramente economicista, la autora pone en primer plano el contexto de economía abierta de competencia internacional y los intentos por adecuar la educación a sus desafíos. El conjunto de requerimientos de la sociedad del conocimiento a nivel global se subraya,

obliga a aumentar el potencial científico-tecnológico de las naciones, a generar capacidades y destrezas indispensables para la competitividad internacional y a desarrollar estrategias para formar una moderna ciudadanía vinculada a la competitividad de los países, a la democracia y la equidad (GAJARDO, 2012, p. 5).

Además, se señala el importante rol del Estado como "garante de equidad", pero también se promueve la intervención del sector privado y el traslado de costos a las familias. El texto apela, como estrategia de argumentación, a lo que "la experiencia internacional" y "la evidencia" "muestran" o "enseñan", sin especificar muchas veces 
qué casos o estudios se toman en cuenta. El análisis de las tendencias de política de la región se ilustra con numerosos ejemplos de países, o incluso de políticas subnacionales en países federales, como Brasil.

El documento identifica cuatro grandes ejes de política desde comienzos de la década de 1990 hasta fines de los 2000: “i) cambios institucionales y de reorganización del sistema escolar, ii) mejorías de calidad y equidad, iii) evaluación de logros de aprendizaje y creación de mecanismos de rendición de cuentas, y iv) desarrollo profesional docente" (GAJARDO, 2012, p. 5). Respecto al primer punto, el balance tiende a ser desalentador. La descentralización de la gestión, aunque tuvo efectos positivos en algunos casos en términos de eficiencia administrativa, aumentó las diferencias de calidad y no contribuyó a reducir desigualdades sociales ni a la mejora general de los aprendizajes. Las políticas de reorganización institucional y mayor autonomía escolar muchas veces no estuvieron acompañadas de apoyos centrales adecuados, coordinación efectiva entre los distintos niveles y de sistemas de compensación para las jurisdicciones y escuelas en situación desventajosa, si bien se rescatan algunos casos en los que hubo efectos positivos derivados de un mayor acercamiento a las escuelas.

Según el documento, en cambio, las reformas para mejorar la calidad y equidad en las escuelas han tenido más éxito. Se destacan, en este sentido, aspectos positivos en la focalización de los programas hacia grupos vulnerables y su aceptación como una política que acierta en lo que se espera sea el rol del Estado como garante de la equidad. Por otra parte, la visión positiva que se transmite sobre el modelo de los países centroamericanos (con una alta autonomía escolar, participación de la comunidad y transferencia de costos a los padres) podría considerarse como contradictoria con lo señalado para el primer punto sobre los efectos nocivos o nulos de los cambios institucionales.

En el punto III, en tanto, se enfatiza el establecimiento de sistemas de medición de logros de aprendizaje, aunque requieren ajustes para contribuir al mejoramiento de la calidad, así como el interés de los países por participar en evaluaciones internacionales. Sobre el trabajo docente, finalmente, se señala "una tendencia hacia la mejoría de las condiciones del trabajo y su profesionalización" (GAJARDO, 2012, p. 16).

Además, se remarcan otros aspectos en el escenario de las reformas: la concreción de acuerdos nacionales, nuevas leyes y marcos normativos que facilitarían "los cambios institucionales y la formulación de políticas de mediano y largo plazo" (GAJARDO, 2012, p. 16); avances en la formulación de diagnósticos más precisos, 
aunque no tanto en las estrategias para abordar los problemas identificados; y el aumento del gasto público, si bien no ha crecido la inversión por alumno y no se alcanza en ningún país el porcentaje recomendado del PIB para "garantizar equidad, calidad y eficiencia educativa” (GAJARDO, 2012, p. 16).

El gran desafío pendiente en la región es lograr "una escuela pública de calidad para todos" (GAJARDO, 2012, p. 16). Se enfatiza la necesidad de nuevas evaluaciones sobre las ventajas y desventajas de los modelos de gestión descentralizada y acerca de su impacto sobre los actores del sistema o su incidencia sobre la calidad de los aprendizajes y la eficiencia escolar. Con relación a la universalización de la cobertura y mejoramiento de los logros de aprendizaje, se mencionan graves dificultades para los países de menor desarrollo e ingresos bajos (como Haití, Nicaragua y Guatemala) en el nivel primario. Para los países del Cono Sur, en cambio, el mayor desafío de cobertura se encuentra en el nivel secundario y en incluir a los excluidos, reducir desigualdades y mejorar los logros de aprendizaje en todos los niveles, sobre todo en los sectores pobres. En esta línea se destaca la necesidad aún de mayor inversión en políticas destinadas a dichas cuestiones.

Las recomendaciones del documento se centran en medidas que ayuden a los estudiantes a aprender mejor y a los docentes a enseñar mejor, así como a mejorar la eficiencia de los sistemas escolares. También se mencionan la necesidad de transformaciones curriculares de fondo y la de invertir en la creación de comunidades de aprendizaje y redes de información y comunicación que faciliten la interacción entre actores sociales y niveles diversos de decisión. El fortalecimiento de la docencia aparece como un punto clave, en que se sugiere mejorar salarios y adaptar políticas de países que reclutan a los docentes en forma más selectiva y que han desarrollado enfoques más efectivos de capacitación. También elevar el gasto por alumno, acompañado por un uso más eficiente y equitativo de los recursos, priorizando la educación básica y los sectores de menores recursos, e incluyendo el financiamiento de las familias y los aportes privados.

El tercer texto (POGGI, 2014) es el documento básico del X Foro Latinoamericano de Educación -organizado por la Fundación Santillana con el patrocinio de la OEI y escrito por quien se desempañaba en ese momento como directora del Instituto Internacional de Planificación Educativa (IIPE/UNESCO, Buenos Aires). Titulado "La educación en América Latina: logros y desafíos pendientes", se enfoca en el análisis de los sistemas educativos y las políticas de inclusión en términos de acceso y mejora de los 
aprendizajes. Desde una perspectiva sistémica, aborda la definición de estrategias macro y micropolíticas y señala la necesidad de (re) diseñar intervenciones de corte universalista.

El documento pone de manifiesto la compleja relación entre democracia, justicia y educación. En esta clave y recuperando la perspectiva de la UNESCO, concibe la educación como derecho individual y social en relación directa con el rol del Estado. Ofrece una discusión teórica sobre la inclusión, a la cual incorpora la cuestión de la calidad de los aprendizajes como "dimensiones inseparables". En forma complementaria, se remite al concepto de democratización de los sistemas educativos, cubriendo sus dimensiones cuantitativa (ampliación del acceso en cantidad de personas/grupos y de años) y cualitativa (disminución de las desigualdades o brechas en las tasas de acceso de los alumnos).

Se presenta un balance favorable del rol que han desempeñado las políticas de inclusión con avances, especialmente, en el acceso, permanencia y finalización de los distintos niveles (inicial, primaria, secundaria) acompañados de una disminución considerable de desigualdades entre estratos socioeconómicos. Asimismo, se destacan logros más claros en la dimensión cuantitativa que en la cualitativa de la democratización. También se mencionan aspectos positivos en la alfabetización de adultos y jóvenes, la paridad de género y el mejoramiento del financiamiento. Sobre el nivel secundario, se señala el efecto positivo de las políticas de ampliación de la obligatoriedad en los 90, ampliadas en la década de 2000 a través de cambios en la normativa y el incremento de la oferta. No obstante, se remarca que existen diferencias importantes entre países respecto a los efectos democratizadores de las políticas. Se valoriza positivamente la orientación de la agenda regional en la búsqueda de igualdad sin ignorar la diversidad geográfica y cultural de cada país.

Poggi (2014) distingue entre dos tipos de políticas de inclusión:

a) las políticas de protección social inclusiva que se manifiestan en los Programas de Transferencias Condicionadas y que se implementan en casi todos los países de la región. Sus efectos en la escuela media han implicado mayores avances en aquellos países que partían de logros educativos menores (menciona Colombia, Ecuador y Paraguay);

b) políticas de mejora de los aprendizajes. Existen dos tipos de estrategias, que en algunas ocasiones se combinan:

- apoyos institucionales externos a través de mecanismos de asistencia técnica, que muchas veces forman parte de programas o proyectos de mejora ${ }^{8}$ de la gestión institucional y de la enseñanza; 
- fortalecimiento de capacidades internas de instituciones y actores escolares ${ }^{9}$ con acciones de desarrollo profesional. Más que en una evaluación de los resultados de estas iniciativas (se aduce poco tiempo de implementación), el texto se detiene en sus ventajas y sus riesgos potenciales.

Los mayores desafíos pendientes son la ampliación de la oferta en la educación inicial y en la secundaria, y la mejora de las trayectorias escolares en educación primaria. En secundaria, además, la finalización del nivel secundario para los estudiantes más vulnerables que lleve a la reducción de las brechas sociales y geográficas. Estos son desafíos que se acentúan para las poblaciones indígenas y afrodescendientes. Los países de la región, se argumenta, deben avanzar en la generación de condiciones y en la formación específica para fortalecer las capacidades de los actores -supervisores e inspectores escolares- que desempeñan un rol fundamental en la institución (POGGI, 2014, p. 70).

Se recomienda reformular ciertos principios de las políticas educativas, pasando del universalismo homogeneizador al reconocimiento de la diversidad de identidades. También manifiesta la necesidad de seguir interviniendo sobre ciertos mecanismos de selección al interior de las escuelas que resultan más injustos porque no se explicitan. El documento plantea "cambiar representaciones y modificar prácticas que conduzcan a dar forma a trayectorias escolares más pertinentes para el conjunto del alumnado" (POGGI, 2014, p. 71). Esto, desde una aproximación sistémica, implica el desafío de alcanzar un conjunto de escuelas de un sistema, no sólo algunas. También apunta la necesidad de coherencia entre las finalidades, los propósitos de mejora educativa y las estrategias que se promueven.

El cuarto documento por considerar (BELLEI, 2013) fue elaborado por académicos de la Universidad de Chile para la OREALC/UNESCO. Se trata de un diagnóstico cuantitativo y cualitativo de la evolución de los sistemas educativos de la región en el período 2000-2010, con relación a las metas 2015 de Educación Para Todos, fijadas en 2000. Además de caracterizar la situación educativa, el texto se propone discutir los desafíos emergentes para la agenda educativa post $2015 .{ }^{10}$ Con la calidad ${ }^{11}$ como tema organizador, se abordan todos los niveles educativos, incluyendo el superior.

Con un enfoque similar a Poggi (2014), el texto plantea una concepción del derecho a la educación que incluye la escolaridad, el aprendizaje y la igualdad de oportunidades. Asimismo, se destacan elementos del contexto de la década de 2000, incluyendo la persistencia -a pesar de un proceso de crecimiento económico y de disminución 
de la pobreza- de elevados niveles de inequidad y pobreza, ${ }^{12}$ y la alta proporción de ruralidad, los cuales habrían ofrecido "dificultades a la expansión de una educación de calidad en la mayor parte de la región” (BELLEI, 2013, p. 22).

El balance de la evolución a lo largo de la década indica que la región realizó importantes avances, pero que subsisten diferencias significativas entre países, así como al interior de la mayoría de los países. Las desigualdades internas, calificadas como "agudas", están asociadas, principalmente, a la clase social, a la condición indígena y a la zona de residencia. En particular, la región exhibe un déficit general en los logros de aprendizaje. A ello se le suma la "persistencia de patrones discriminatorios tanto en términos culturales como pedagógicos e institucionales" (BELLEI, 2013, p. 29), que afectan especialmente a los estudiantes de menores recursos y de poblaciones indígenas. Por otra parte, se señala que el aumento de la inversión educativa ha acompañado el crecimiento de los sistemas educativos, pero no ha llegado a modificar significativamente el gasto por alumno.

Al mismo tiempo, se destacan algunas políticas exitosas, por ejemplo, en primaria, los programas de transferencias condicionadas -aunque se señala que debería mejorarse su dimensión educativay la prolongación de la jornada escolar (casos de Chile, Colombia y Uruguay). En el nivel secundario, programas preocupados por la equidad socioeducativa, como el Liceo para Todos (Chile). Dentro de las políticas docentes, se menciona el Fondo de Manutención y Desarrollo de la Educación Primaria y de Valorización del Magisterio (FUNDEF) de Brasil, que habría permitido mejorar los salarios y destinar fondos a la capacitación docente.

Los principales desafíos que el documento señala respecto a la educación básica son:

a) lograr una mayor homogeneidad en cuanto a la cobertura y la calidad de la educación preprimaria;

b) proveer mejores condiciones para que los niños y adolescentes en situación de mayor desventaja social no deserten de la escuela primaria y adquieran las competencias necesarias para progresar a la secundaria;

c) romper con la fuerte segregación socioeconómica del sistema educativo que implica escuelas de baja calidad para los más pobres;

d) configurar una carrera profesional docente que atraiga a los jóvenes talentosos, forme adecuadamente, retenga en las aulas a los buenos profesores y jerarquice el desarrollo profesional; 
e) promover una "sana convivencia" entre estudiantes y docentes, mediante la participación estudiantil y "superando los enfoques puramente punitivos de control de la violencia y disciplinamiento" (BELLEI, 2013, p. 26);

f) reponer la formación ciudadana como un componente central del currículo y de la calidad de la educación.

Ante estos desafíos, el documento sugiere centrar esfuerzos en remover las barreras (costos, repitencia y deserción) para una educación primaria para todos. En contraste con algunas de las propuestas de Gajardo (2014), critica la idea de expandir la educación privada por sus efectos segmentadores. Además, plantea la necesidad de una "reforma integral" de la escuela secundaria para combatir las altas tasas de abandono y para que los jóvenes encuentren el sentido de este nivel educativo; a la vez, propone una integración más efectiva con la formación para el trabajo. También se sostiene que las capacidades docentes son "el pilar fundamental de la calidad educativa" (BELLEI, 2013, p. 25), en coincidencia con la importancia que los demás documentos analizados le asignan al factor docente.

Otras recomendaciones se orientan a la generación de "mejores oportunidades de aprendizaje para todos" (BELLEI, 2013, p. 25) y en especial para los alumnos con dificultades, poniendo los sistemas de evaluación de aprendizajes al servicio de dicha mejora; el impulso a un enfoque de formación ciudadana que se articule a la formación académica y que habilite para la participación ciudadana y política; y la promoción de la educación intercultural para todos, y de la educación intercultural bilingüe para las poblaciones indígenas, con los recursos materiales y humanos adecuados.

En quinto lugar, consideraremos en forma conjunta dos Informes de la OEI $(2014 ; 2013)$ que buscan medir los avances en el logro de las "Metas 2021" establecidas por los países de la región para sus sistemas educativos en forma consensuada entre el 2008 y el 2010. El de 2014 fue el primer informe completo del progreso en el cumplimiento de las metas en conjunto y en cada uno de sus indicadores, mientras que el de 2013 se concentró en la situación, la formación y la evaluación del profesorado en la región, con énfasis en los casos de México y Uruguay. ${ }^{13}$

En base al planteamiento general del documento que explicita las Metas 2021 (SEGIB/OEI/CEPAL, 2010), podría sostenerse que los textos analizados responden a una perspectiva ecléctica que promueve un tipo de cambio educativo que responda tanto a los imperativos de la economía global como a objetivos de democratización e igualdad 
social. Uno de los supuestos fundamentales de estos informes es que "la educación constituye una estrategia fundamental para avanzar en la cohesión y la inclusión social en Iberoamérica" (OEI, 2014, p. 12). A la vez, se propone como horizonte "un sistema educativo inclusivo, con calidad y pertinencia y orientado a la transmisión de conocimientos científicos y de saberes ancestrales y a la promoción de la convivencia en un marco de valores democráticos y éticos" (OEI, 2013, p. 9). Calidad, equidad e inclusión son conceptos clave en la explicitación de objetivos a nivel sistémico en ambos documentos. El tono general es descriptivo, con pocas alusiones a valoraciones de las políticas implementadas.

Sin embargo, se trasluce un balance general positivo: "la educación ha progresado enormemente en todos los países y se ha convertido en uno de los objetivos prioritarios en las políticas públicas de la mayoría de ellos" (OEI, 2014, p. 7). Se destacan aumentos en la inversión, mejoras en las tasas de finalización en primaria y secundaria, el descenso del analfabetismo, las políticas de jornada escolar completa (El Salvador, Honduras y República Dominicana), nuevos proyectos para la atención integral de la infancia. No obstante "continúan manifestándose claras diferencias y desigualdades entre unos países y otros y dentro de cada país en la mayoría de los indicadores seleccionados" (OEI, 2014, p. 8). Se señalan problemas de equidad en la cobertura, bajos logros de aprendizaje, déficits en infraestructura escolar e insuficiente tiempo dedicado a la enseñanza, así como la necesidad de avanzar más hacia una educación inclusiva e intercultural, en la que se garantice el pleno derecho a la educación de los grupos originarios y afrodescendientes.

En lo referido al profesorado, se destacan tanto iniciativas innovadoras como prácticas tradicionales, que en algunos casos conspiran contra los objetivos de una educación inclusiva y de calidad. Como aspectos positivos, se mencionan la instauración de sistemas de acreditación de instituciones formadoras y sus programas; de pruebas de egreso o habilitación para el ejercicio profesional; la formulación de estándares y lineamientos para orientar los currículos y procesos evaluativos de las instituciones formadoras; y el fortalecimiento de las relaciones entre instituciones formadoras y escuelas de educación básica. Al mismo tiempo, como desafíos se identifican: hacer la profesión docente más atractiva para que accedan a ella los jóvenes mejor preparados y motivados; generar modelos de formación inicial y permanente actualizados y que respondan a las necesidades de los estudiantes; y atender la complejidad que genera la inclusión y la universalización. 
Las principales recomendaciones referidas a las políticas docentes, varias de ellas similares a las formuladas en el texto de Bellei (2013), se centran en:

a) mejorar la atracción, selección, formación y retención;

b) favorecer el diálogo entre las administraciones educativas y los sindicatos docentes, y entre las instituciones formadoras de docentes y las escuelas;

c) proponer un sistema de evaluación docente que tenga como fin último mejorar los aprendizajes de los estudiantes y que sea parte de un sistema integral de evaluación del sistema educativo;

d) implementar modelos de formación que garanticen la utilización de metodologías flexibles del aprendizaje y de estrategias de innovación, la incorporación de las TIC y la reflexión sobre la propia práctica docente;

e) plantear una mayor diferenciación en la formación continua.

El documento incluye una propuesta muy detallada y fundamentada de los rasgos que debería presentar un sistema de evaluación docente que tenga como fin último mejorar los aprendizajes de los estudiantes y que sea parte de un sistema integral de evaluación del sistema educativo.

La comparación entre los documentos analizados permite identificar una serie de convergencias y divergencias. Entre las primeras, aparece la idea de que los sistemas educativos en la región han actuado como reproductores de la desigualdad social, aunque este planteo no se acompañe de un análisis de las causas de la pobreza y la desigualdad. La ausencia de cuestionamientos al orden social o económico, que es característico de estos textos, implica que es necesario y suficiente un ajuste interno de los sistemas educativos para convertirse en herramientas que contribuyan a una sociedad más equitativa.

Otro de los elementos comunes es el énfasis tanto en la cobertura como en la mejora de los aprendizajes, y en la necesidad de enfocar esfuerzos en la atención a las poblaciones más postergadas. Ya en la nueva generación de leyes educativas que emergía en la primera mitad de los años 2000, se había puesto de manifiesto la urgencia de considerar el problema del acceso con relación al conocimiento y no meramente a la escolaridad (LÓPEZ, 2007). Por otra parte, Oreja Cerruti y Vior (2016, p. 31), refiriéndose al discurso de tipo social del Banco Mundial y el BID en sus documentos más recientes, argumentan que esta preocupación social puede verse como un "neoliberalismo aggiornado que procura, principalmente, el mantenimiento del orden social en sociedades 
fuertemente desiguales", algo que había sido notado por Bonal (2002) para el caso del Banco Mundial desde fines de la década de 1990.

Finalmente, cabe destacar como otro aspecto de fuerte convergencia la centralidad asignada a las políticas docentes en términos de las recomendaciones para la mejora de los sistemas educativos. Si bien la importancia otorgada al factor docente es compartida por el discurso académico en la región (véase, por ejemplo, VAILLANT, 2013; VEZUB, 2013), temas como los dispositivos de formación continua o las formas de evaluación del trabajo docente están lejos de concitar consensos amplios.

En términos de las divergencias entre los documentos, nos interesa señalar, en primer lugar, que existen significativas diferencias en las perspectivas de análisis adoptadas, desde enfoques humanistas a miradas de tipo economicista, pasando por visiones más eclécticas o mixtas. Esos diferentes lugares desde donde los textos abordan las políticas educativas tienen claros correlatos -más allá de los puntos comunes discutidos en los párrafos anteriores- en términos del tipo de balance que realizan y de las recomendaciones que plantean. Uno de los puntos en los que se pone más claramente de manifiesto dichos correlatos es en la cuestión de la promoción de la privatización que, a diferencia del panorama que presenta Krawczyk (2002) para el período 1998-2001, aquí aparece como un elemento algo marginal en el conjunto de los textos ${ }^{14}$ y explícitamente rechazado en Bellei (2013). Otros son los del énfasis en la democratización de los sistemas educativos, o el del fortalecimiento de la educación intercultural, aspecto que cobra un lugar protagónico en algunos de los documentos mientras que es ignorado por otros, y que, como señalamos anteriormente, parece haber ganado prioridad en las leyes recientemente sancionadas en la región (SAFORCADA; VASSILIADES, 2011).

Hay otras dos cuestiones en las que surgen importantes diferencias de posicionamiento respecto a las sugerencias de política. La primera se relaciona con el grado de homogeneidad de las recomendaciones: mientras que en algunos casos -Cabrol y Székely (2012) representan la postura más extrema en este sentido- se pretende identificar políticas universalmente válidas, lo cual ha sido objeto de una crítica repetida a los enfoques de los organismos internacionales (CORAGGIO; TORRES, 1997), en otros documentos se promueve una mayor diferenciación de las políticas en términos de la situación heterogénea de los sistemas educativos nacionales o de las necesidades y demandas de distintos grupos sociales a su interior. El segundo elemento se refiere al foco y priorización entre niveles educativos. Si bien hay una cierta tendencia a 
destacar la necesidad de mejorar la cobertura del nivel inicial y del tramo superior del secundario, es posible también identificar algunos matices respecto a qué nivel -y en qué medida- privilegiar.

\section{CONCLUSIONES}

Los principales resultados de nuestro análisis apuntan a significativas diferencias de enfoque en los distintos textos, desde perspectivas economicistas hasta visiones humanistas, que se reflejan en diagnósticos y balances con ciertas divergencias. A su vez, las recomendaciones de política varían en los focos, desde la promoción de la diversidad cultural y la democratización de los sistemas educativos, a la expansión del nivel inicial o al mejoramiento de la formación docente. A pesar de eso, hay una tendencia común a identificar la superación de las fuertes desigualdades sociales y la necesidad de políticas que pongan el foco en el aprendizaje como prioridades del trabajo de gobiernos y organismos internacionales.

A diferencia de lo que identificamos como una tendencia en la mayor parte de los estudios previos sobre las propuestas de política educativa de los organismos internacionales para América Latina, en nuestro análisis hemos buscado remarcar divergencias entre los documentos analizados. Aunque también hemos identificado algunos puntos de convergencia importantes, esas diferencias muestran una diversidad de posicionamientos respecto a algunos aspectos significativos de la agenda de reforma educativa. En conjunto, el análisis de los documentos seleccionados permite apreciar cómo se construyen algunas perspectivas de análisis y ejes conceptuales que dan forma al debate actual sobre los logros y desafíos pendientes de los sistemas educativos de la región. Además de contribuir a entender cómo se estructura el discurso de la reforma educativa, un ejercicio de este tipo -como apunta Krawczyk (2002)- brinda indicios de qué temas tuvieron y continuarán teniendo apoyo de estas agencias. Si bien los organismos internacionales son actores que influyen fuertemente en los procesos de formulación de las políticas, vale tener presente que estos procesos, incluyendo sus aspectos discursivos, están constantemente abiertos a cuestionamientos, contestaciones y redireccionamientos por parte del resto de los actores que participan en la tarea educativa. Más aun, como hemos intentado mostrar, ni en conjunto ni en los textos individuales, el discurso de estos organismos es uniforme o unívoco.

Consideramos que sería provechoso abordar, en futuras investigaciones, el análisis de las continuidades y rupturas en el 
discurso de las agencias internacionales entre la década de 1990 y el período post-2000, alrededor del cual varios analistas discuten el alcance del concepto de "postneoliberalismo" o en qué medida ha surgido un nuevo paradigma de políticas educativas, así como de la visión de los propios organismos sobre la relación de las políticas recientes con las reformas implementadas en los noventa. Estos abordajes, a la vez, podrían complementarse con estudios sobre los balances que otros actores (académicos, organizaciones sindicales, gobiernos, etc.) hacen de las políticas que se llevaron adelante en el período y sobre las proyecciones que hacen hacia el futuro.

\section{REFERENCIAS}

BALL, S. Política social y educativa, empresa social, hibridación y nuevas comunidades discursivas. Propuesta Educativa, Buenos Aires, v. 2, n. 36, p. 25-34, 2011.

BELLEI, C. (Coord.). Situación educativa de América Latina y el Caribe: hacia la educación de calidad para todos al 2015. Santiago de Chile: Oficina Regional de Educación para América Latina y el Caribe (OREALC/UNESCO), 2013.

BONAL, X. Globalización y política educativa: un análisis crítico de la agenda del Banco Mundial para América Latina. Revista Mexicana de Sociología, Ciudad de México, v. 64, n. 3, p. 3-35, jul./sep. 2002.

BURCH, P. Hidden Markets: the new education privatization. Abingdon: Routledge, 2009.

CABROL, M.; SZÉKELY, M. Educación para la transformación. New York: BID, 2012.

CEPAL-UNESCO. Educación y conocimiento: eje de la transformación productiva con equidad. Santiago de Chile: CEPAL-UNESCO, 1992.

CORAGGIO, J. L.; TORRES, R. M. La educación según el Banco Mundial: un análisis de sus propuestas y métodos. Buenos Aires: Miño y Dávila Editores/CEM, 1997.

FELDFEBER, M. La regulación de la formación y el trabajo docente: un análisis crítico de la "agenda educativa" en América Latina. Educação \& Sociedade, Campinas, v. 28, n. 99, p. 444-465, maio/ago. 2007.

GAJARDO, M. La educación tras dos décadas de cambio: ¿Qué hemos aprendido? ¿Qué debemos transformar?. Santiago de Chile: PREAL Publicaciones, 2012. (Serie Documentos, n. 65.). Disponible en: <http://educacion.udd.cl/files/2015/04/La-educaci\%C3\%B3n-tras-dosd\%C3\%A9cadas-de-cambio.pdf $>$. Consultado el: 18 feb. 2016.

GOROSTIAGA, J. M.; TELLO, C. G. Globalización y reforma educativa en América Latina: un análisis inter-textual. Revista Brasileira de Educação, Brasília, v. 16, n. 47, p. 363-388, maio/ago. 2011. GUEST, G.; MACQUEEN, K.; NAMEY, E. Applied thematic analysis. Los Angeles: Sage, 2011. KRAWCZYK, N. La reforma educativa en América Latina desde la perspectiva de los organismos multilaterales. Revista Mexicana de Investigación Educativa, Ciudad de México, v. 7, n. 16, p. 627-663, sep./dic. 2002.

KROTSCH, P. Educación superior y reformas comparadas. Bernal: Universidad Nacional de Quilmes, 2001.

LÓPEZ, N. Las nuevas leyes de educación en América Latina: una lectura a la luz del panorama social y educativo de la región. Buenos Aires: IIPE - UNESCO, 2007. 
MARCELO, G. Perspectivismo e Hermenêutica. Impulso, Piracicaba, v. 24, n. 59, p. 51-64, jan./abr. 2014.

MUNDY, K. Educational multilateralism and world (dis)order. Comparative Education Review, Chicago, v. 42, n. 4, p. 448-478, Nov. 1998.

NUDZOR, H. What is "policy", a problem: solving definition or a process conceptualisation? Educational Futures, Londres, v. 2, n. 1, p. 85-96, sept. 2009.

ORGANIZACIÓN DE ESTADOS IBEROAMERICANOS PARA LA EDUCACIÓN, LA CIENCIA Y LA CULTURA. Miradas sobre la educación en Iberoamérica: avances en las Metas Educativas 2021. Madrid: OEI, 2014.

- Miradas sobre la educación en Iberoamérica: desarrollo profesional docente y mejora de la educación. Madrid: OEI, 2013.

OREJA CERRUTI, M. B.; VIOR, S. E. La educación y los Organismos Internacionales de crédito: préstamos y recomendaciones para América Latina (2000-2015). Journal of Supranational Policies of Education, Madrid, n. 4, p. 18-37, 2016.

ORJUELA, L. J. La compleja y ambigua repolitización de América Latina. En: MÁRQUEZ RESTREPO, M. L.; PASTRANA BUELVAS, E.; HOYOS VÁSQUEZ, G. (Ed.). El eterno retorno del populismo en América Latina y el Caribe. Bogotá: Editorial Pontificia Universidad Javeriana, 2012. PEREIRA, J. M. M.; PRONKO, M. A. (Org.). A demolição de direitos: um exame das políticas do Banco Mundial para a Educação e a Saúde (1980-2013). Rio de Janeiro: Escola Politécnica de Saúde Joaquim Venâncio - Fundação Oswaldo Cruz, 2015.

POGGI, M. La educación en América Latina: logros y desafíos pendientes: documento básico. Buenos Aires: Santillana, 2014

PULIDO CHAVES, O. Sobre el derecho a la educación en América Latina. In: PRE-ASAMBLEA LATINOAMERICANA DE LA CAMPAÑA MUNDIAL POR EL DERECHO A LA EDUCACIÓN, 2010. Disponible en: < http://educacionenelmundo.wordpress. com/2010/12/03/analisis-sobre-el-derecho-a-laeducacion-en-america-latina $>$. Consultado el: 25 nov. 2014.

RAMBLA, X. Las complejas geografías de la política educativa: tres estudios de caso. Educaşão \& Sociedade, Campinas, v. 34, n. 125, p. 1229-1249, out./dez. 2013.

RIZVI, F.; LINGARD, B. Politicas educativas en un mundo globalizado. Madrid: Morata, 2013.

RODRÍGUEZ-GÓMEZ, R.; ALCÁNTARA, A. Multilateral agencies and higher education reform in Latin America. Journal of Education Policy, Londres, v. 16, n. 6, p. 507-525, 2001.

SAFORCADA, F.; VASSILIADES, A. Las leyes de educación en los comienzos del siglo XXI: del neoliberalismo al postconsenso de Washington en América del Sur. Educação \& Sociedade, Campinas, v. 32, n. 115, p. 287-304, abr./jun. 2011.

SÁNCHEZ CERÓN, M. Influencia del Banco Mundial y la CEPAL en las tendencias educativas recientes en algunos países latinoamericanos. Revista Latinoamericana de Estudios Educativos, México, D.C., v. 31, n. 4, p. 55-97, 2001

SECRETARÍA GENERAL IBEROAMERICANA - SEGIB; ORGANIZACIÓN DE ESTADOS IBEROAMERICANOS PARA LA EDUCACIÓN, LA CIENCIA Y LA CULTURA - OEI; COMISIÓN ECONÓMICA PARA AMÉRICA LATINA - CEPAL. Metas Educativas 2021: la educación que queremos para la generación de los bicentenarios. Madrid: OEI, 2010. Disponible en: < http://www.oei.es/metas2021/libro.htm>. Consultado el: 5 marzo 2016.

TELLO, C.; MAINARDES, J. A educação secundária na América Latina como um direito 
democrático e universal: uma análise de documentos do Banco Mundial e do Banco Interamericano de Desenvolvimento. Educação e Filosofia, Uberlândia, v. 28, n. especial, p. 155-179, 2014.

VAILLANT, D. Las políticas de formación docente en América Latina: avances y desafíos pendientes. In: POGGI, Margarita (Coord.). Políticas docentes. Buenos Aires: Instituto Internacional de Planeamiento de la Educación IIPE-Unesco, 2013.

VERGER, Antoni. Why Do Policy-Makers Adopt Global Education Policies? Toward a Research Framework on the Varying Role of Ideas in Education Reform. Current Issues in Comparative Education, New York, v. 16, n. 2, p. 14-29, 2014.

VEZUB, L. Hacia una pedagogía del desarrollo profesional docente: modelos de formación continua y necesidades formativas de los profesores. Páginas de Educación, Montevideo, v. 6, n. 1, p. 97-124, jun. 2013

\section{NOTAS}

${ }^{1}$ Esto no significa que estos tres organismos no hayan tenido influencia sobre el discurso y los procesos de formulación de políticas educativas en el período, sea a través de asistencia técnica o financiamiento de proyectos, de diagnósticos y recomendaciones para países particulares o de lineamientos de reforma a nivel global.

${ }^{2}$ Publicado originalmente en MARCHESI, A.; TEDESCO, J. C.; COLL, C. (Coords.). Calidad, equidad y reformas en la enseñanæa. Madrid: OEI, 2009.

${ }^{3}$ Verger (2014), refiriéndose en particular al rol que se les asigna a las ideas globales en los procesos de adopción de políticas públicas, distingue entre una posición constructivista, una racionalista y una institucionalista.

${ }^{4}$ Surgida como un producto de la Cumbre de las Américas, se conformó a partir de una red de organizaciones públicas y privadas gestionada en forma conjunta por el Inter-American Dialogue y la Corporación de Investigaciones para el Desarrollo (CINDE, Chile).

${ }^{5}$ El trabajo consideró las publicaciones del Banco Mundial, la CEPAL, PREAL, el BID, el IIPE/UNESCO y OREALC/UNESCO desde 1998 hasta 2001.

${ }^{6} \mathrm{El}$ análisis se basa en dos capítulos: "Introducción: ¿Cómo lograr una educación para la transformación?” y "Educación para la transformación: conclusiones"

${ }^{7}$ Sorprende -por lo incorrecta- una afirmación en la que la clásica caracterización de "década pérdida" es atribuida a los 90 en lugar de los 80 y en la que se ignora, a la vez, la magnitud y el impacto de la ola de reformas educativas de los años 90 en la región: "En América Latina y el Caribe (ALC) la agenda educativa pasó a segundo plano durante las últimas dos décadas del siglo XX (incluida la 'década perdida' de 1990)”. (CABROL; SZÉKELY, 2012, p. v).

${ }^{8}$ Ejemplos: el Programa de Escuelas de Calidad y el Programa Escuelas de Tiempo Completo en México, el Plan de Mejoramiento Educativo vinculado con la Subvención Escolar Preferencial y el Programa Mejor Escuela en Chile, la política de Mejoramiento de las condiciones de enseñanza y aprendizaje en Argentina, el Proyecto de Apoyo a la Educación Secundaria para la Reducción del Abandono Escolar en Costa Rica, el Programa Contemos Juntos en Guatemala, el Programa Pequeños Matemáticos en Paraguay.

9 "Pueden rastrearse políticas y experiencias en países como Argentina, Brasil, Colombia, Chile, Ecuador, México, Perú, Uruguay, entre otros.” (POGGI, 2014, p. 70).

${ }^{10}$ En la "Presentación", el director de la OREALC destaca que el documento busca contribuir a "la nueva agenda educativa". 
11 "La concepción de calidad que aplicamos es amplia e incluye no solo logros, sino condiciones y procesos, no solo aspectos académicos, sino psicosociales y ciudadanos." (BELLEI, 2013, p. 17).

${ }^{12}$ Se muestra que el coeficiente Gini de distribución del ingreso sólo se redujo de aproximadamente 0,53 a 0,51 entre 2000 y 2010.

${ }^{13}$ En este caso se analiza en particular el capítulo 6, "Conclusiones y propuestas de mejora". En el documento no se aclara por qué fueron elegidos los casos de México y Uruguay.

${ }^{14}$ Otros análisis sobre el posicionamiento del Banco Mundial y del BID en las reformas educativas de la región, sin embargo, identifican la defensa de la privatización a través de formas más o menos encubiertas (OREJA CERRUTI; VIOR, 2016; TELLO; MAINARDES, 2015).

Submetido: $13 / 09 / 2016$

Aprovado: 02/02/2017

Contato:

Jorge M. Gorostiaga

Escuela de Humanidades (UNSAM) Martín de Irigoyen 3100, San Martín

Prov. de Buenos Aires, Argentina

Código postal: 1650 